\title{
El efecto de la educación en el crecimiento económico: el caso de México en el periodo 1996-2016
}

\section{Faustino Vega Miranda ${ }^{1}$}

\section{Resumen}

El objetivo de la investigación es determinar el tipo de relación que existe entre el gasto en educación y el crecimiento económico de la economía mexicana en el periodo 1996-2016. Para la teoría del capital humano, la relación educación-crecimiento es directa y clara, sin embargo, desde una perspectiva empírica, en los países en vías de desarrollo están presentes ciertas características que permiten inferir una correlación negativa o la inexistencia de dicho efecto. Para ello se determinó la prueba de causalidad en el sentido de Granger y la evidencia registra independencia entre las dos variables analizadas, por lo que el enfoque del capital humano no es universal.

Palabras clave: Educación, Crecimiento Económico, Capital Humano, Causalidad, Organismos Internacionales.

Clasificación JEL: I20, F43, J2, B23, O19.

\begin{abstract}
The objective of the research is to determine the type of relationship that exists between spending on education and economic growth for the Mexican economy in the 1996-2016 period. For the theory of human capital, the relationship education-growth is direct and clear, however, from an empirical perspective, in the developing countries are present certain characteristics that allow to infer a negative correlation or the

1 Profesor adscrito a la Licenciatura en Economía en la Facultad de Estudios Superiores Acatlán (fvega@comunidad.unam.mx).

* Fecha de recepción: 22/01/2018. Fecha de aceptación: 23/01/2019.
\end{abstract}


inexistence of this effect. To do this, the causality test was determined in the sense of Granger and the evidence registers independence between the two variables analyzed, so that the approach of human capital is not universal.

Keywords: Education, Economic Growth, Human Capital, Causality, International Organizations.

JEL Classification: I20, F43, J2, B23, O19.

\section{Introducción}

Para los economistas el estudio de la relación educación-crecimiento es clara y simple desde la perspectiva del capital humano, en la práctica esta teoría sustenta las recomendaciones de los principales organismos internacionales, es decir, dota de financiamiento a los distintos sectores de un sistema educativo.

A partir de una perspectiva crítica, los pedagogos no están totalmente de acuerdo con tales postulados, niegan la universalidad del enfoque utilitarista con base en investigaciones empíricas; en países en vías de desarrollo la asignación del gasto educativo es discrecional, la corrupción es una variable constante en los diferentes gobiernos y el desempleo afecta a la mayoría de la población, en especial a los profesionistas. Educación no es sinónimo de movilidad social y la relación directa que postulan los teóricos del capital humano sólo queda impresa en los libros de macroeconomía.

Entonces, si la teoría no es universal y no tiene sustento en la realidad contemporánea, por qué se sigue empleando dicho enfoque para explicar las relaciones entre educación y crecimiento. La respuesta se encuentra en la influencia de esta teoría sobre la política y en el pensamiento público. Los organismos internacionales han encontrado en la narrativa de la teoría una explicación simple basada en la idea de una relación lineal entre educación y crecimiento. También es cierto que algunas instituciones han retomado sus principios en otro sentido, el 
Banco Mundial (BM) propone la política educativa desde la perspectiva de la enseñanza técnica y educación primaria, mientras la educación terciaria es relegada.

Lauder (2015) propone otra razón, los organismos internacionales han encontrado en el enfoque de capital humano una justificación para el financiamiento de la educación, los préstamos de recursos dirigidos a los distintos países pueden condicionarse debido al capital humano. En 2018 el BM (2018) desarrolló un proyecto para medir el capital humano a través de un índice que puede tomar valores de 0 a 1 , el país refleja mejores condiciones para desarrollar las habilidades de las personas cuando el número sea más cercano a la unidad. Por lo que este indicador perpetua las condiciones del modelo educativo modernizador porque se enfoca en promover la educación básica y excluir la educación superior. Además, el BM promueve implícitamente una política educativa vertical; los países para escalar en el ranking y conseguir apoyo financiero deben mejorar en las pruebas internacionales y esa meta se logra por medio de la implantación total del modelo por competencias. El índice del capital humano puede exacerbar las condiciones de financiamiento hacia los países.

Bajo este contexto, el presente documento busca contribuir al debate académico determinando el tipo de relación que existe entre gasto educativo y crecimiento económico en México en el periodo 1996-2016. Para lograr dicha tarea el artículo se compone de tres secciones. En la primera parte se analiza la narrativa del capital humano, su origen y la crítica de los sociólogos por medio de la cultura. En segundo lugar, se revisan los estudios empíricos sobre la relación educación-crecimiento económico y en la tercera sección se establece la propuesta metodológica que involucra el estudio de la prueba de causalidad en el sentido de Granger, así como la descripción de las variables analizadas en la investigación y la estimación del modelo para el caso de México. El texto finaliza con la exposición de las conclusiones. 


\section{La propuesta de la teoría económica, el enfoque del capital humano}

La investigación de Adam Smith se centró en encontrar las variables que determinaban el crecimiento de una nación, de manera indirecta infirió que la educación juega un papel relevante para reducir los problemas que podrían mostrar las variables que tienen alguna influencia en el crecimiento económico (Galindo y Méndez, 2010). Cabe resaltar que el padre del liberalismo no percibía inadecuado que el gobierno proveyera educación.

David Ricardo no trató el tema de la educación, de hecho, el economista inglés no prestó mucha atención al tema; en la lógica del autor inglés el crecimiento estaba determinado por los factores de la producción (Bouznit, 2012) y por el interés de la ventaja comparativa.

Por mucho tiempo la relación educación-crecimiento se difuminó en la academia, fue hasta la época de la posguerra cuando se revivió el interés. Desde un planteamiento heterodoxo, Harrod (1939) y Domar (1946) encontraron que existe una tasa de crecimiento de la inversión (física e inmaterial) que asegura el pleno empleo del ingreso y de la ocupación. Las posiciones ortodoxas respondieron con la contribución de Solow (1956), su modelo apareció en respuesta al modelo de Domar, bajo cinco supuestos para el desarrollo de la ecuación fundamental (Aghion y Howitt, 2009), donde el crecimiento se ha atribuido a un factor "residual" que representa el progreso técnico o la "calidad del trabajo" que se traducirá por lo siguiente en la concepción de "capital humano" (Okacha, 2015).

Con base en el modelo de Solow, se definió una agenda de investigación sobre la relación educación-crecimiento. Desde una perspectiva micro, para J. Mincer y G. Becker la educación ayuda al aumento de la productividad de las personas y les permite aumentar su stock de capital humano y, por lo tanto, aumentar su salario (Diop, 2011). Inclusive Mincer realizó una serie de estimaciones para cuantificar la tasa de rendimiento escolar (De la Fuente y Ciccone, 2002). 
Mankiw et al. (1992) proponen un modelo donde el producto se determina por tres factores: trabajo, capital físico y capital humano. Una de las conclusiones que se derivan del modelo neokeynesiano tiene que ver con el incremento del gasto en educación para que un país experimente un rápido crecimiento en el nivel de renta del estado estacionario (Weil, 2006), porque la inversión en capital humano genera efectos indirectos que constituyen un beneficio para todo el sistema social en su conjunto (Rajimon, 2010). En estudios prácticos, Barro (1999) encuentra que un año adicional de escolaridad aumenta la tasa de crecimiento del producto en un $0.7 \%$, dentro de un estudio robusto para varios países.

Recientemente, existe una amplia literatura que estudia el impacto del capital humano en el crecimiento económico, Hanushek y Kimko (2000), así como Hanushek y Woessmann (2012) siguen la misma línea argumentativa; encuentran evidencia empírica que valida una relación directa entre dos categorías, cantidad de años de estudio y crecimiento económico. Sin embargo, Valdenaire (2011) encuentra algunas contradicciones entre estudios, lo que lleva a sospechar que los resultados de esta literatura sean realmente válidos. Por lo tanto, actualmente la educación en un elemento primordial en el crecimiento económico dentro de las economías de mercado desde el enfoque del capital humano.

\subsection{La consolidación del enfoque del capital humano, la expansión de EU}

Las habilidades adquiridas en la escuela incrementan la posibilidad de conseguir un empleo, aumentan la productividad y la relación salarios-ganancias crece sin fomentar un problema distributivo. Estas ideas, condensadas en el enfoque del capital humano, son producto de un momento histórico particular, la edad de oro del capitalismo norteamericano. Marginson (2017) ubica este origen en la expansión económica de EU entre las décadas de 1950-1970 que permitió el crecimiento de la educación superior en masa y movilidad social.

No obstante, el origen del concepto se relaciona con la concepción de la educación, con sus funciones. Para Laval (2004) la educación ha 
tenido dos funciones, cultural y económica. La relación fue más o menos equilibrada por la intervención del Estado al proveer de servicio educativo a los grupos más vulnerables. Los hechos se desarrollaron de esta manera hasta acabada la segunda guerra mundial. En la época post bélica, la escuela sufrió un sometimiento por el capital y la función económica se ha exacerbado: la escuela sólo debe enseñar cosas útiles y la enseñanza se cuantifica por medio de símbolos y grados.

Más recientemente, se dio una segunda transformación, la escuela empezó a adoptar métodos de gestión empresarial, la llamada modernización. La función cultural de la educación fue totalmente relegada por la concepción utilitarista; si el conocimiento no tiene una función en la empresa, no sirve. Sin embargo, el capital cultural es tan importante como el capital económico. En la lógica de la transmisión del capital cultural es donde reside el principio más poderoso de la eficacia ideológica de este tipo de capital (Bourdieu, 1987). El capital cultural es primordial para entender de mejor forma las trayectorias escolares (Colorado, 2009) y las condiciones de crecimiento de un país.

Desde esta perspectiva, el origen del capital humano o de la concepción utilitarista se remonta a autores como F. Bacon, R. Spencer y A. Smith, pero su consolidación viene cuando las naciones se estabilizan después de 1945. Cuando la función económica y cultural de la educación se encontraban armonizadas, la teoría del capital humano fungió como una primera y primitiva explicación de la relación educación-crecimiento. El enfoque considera que los salarios se encontraban determinados por las habilidades de los distintos agentes, pero en este periodo en específico. El problema es que este enfoque trascendió y los economistas emplean en la actualidad tal explicación como universal (Lauder, 2015), cuando no es así. Aún más peligroso, los organismos internaciones adaptan esas ideas para emitir recomendaciones de política educativa.

La propuesta del capital humano no es universal, los salarios no exclusivamente responden a las habilidades adquiridas en la escuela, sino que, además, intervienen factores no educativos (meritocracia, selección universitaria, corrupción, condiciones económicas del país, etc.). 
Para Marginson (2017), la teoría neoclásica del capital humano carece de realismo al menos por cuatro razones:

- Es un sistema cerrado, no lo afectan variables externas.

- La narrativa es lineal y simple.

- Une mundos heterogéneos, educación-trabajo, y entre ellas existe una única relación.

- Omite las posibles relaciones disfuncionales entre educación y trabajo.

El inciso dos resalta de inmediato a los investigadores en Pedagogía; a ellos les es difícil entender que la educación se pueda reducir a una regresión lineal simple y que, a través de este instrumento matemático-estadístico, sea posible medir efectos a toda la economía, suprimiendo las características particulares de los diferentes entes que conforman el todo. Además, la respuesta no puede ser universal porque la teoría no explica cómo contribuye la inversión en educación al bienestar de las organizaciones, comunidades y sociedades (Mayia et al., 2004).

Finalmente, una prueba cuantitativa para negar la funcionalidad de la teoría del capital humano es la movilidad social. En la época reciente se ha concentrado la riqueza material en el mundo desarrollado (Piketty, 2014), pero también se ha agudizado la desigualdad en los países en vías de desarrollo. La Organización para la Cooperación y el Desarrollo Económico (OCDE, 2018) reconoce que la movilidad social se ha estancado en las últimas tres décadas, es decir, los ricos y pobres son más propensos a permanecer en esa condición. En México 70 de cada 100 personas que nacen en el quintil más bajo de la distribución del ingreso permanecen ahí (Delajada et al., 2018), o sea, 7 de cada 10 mexicanos nace y muere en condición de pobreza.

\subsection{EI reproche de los otros enfoques, la perspectiva social}

El concepto de capital cultural es un aporte en el campo de la sociología (Marcenaro y López, 2014), es una de las categorías más empleadas por los autores afines a la disciplina, de ahí sus múltiples definiciones (Bra- 
cho, 1990), desde el conocimiento de alta cultura hasta el conocimiento de tecnología como señal de estatus social. En una aproximación general, Bourdieu desarrolló una perspectiva multidimensional por medio del capital cultural que involucra lo económico, cultural, social y simbólico.

Visto a retrospectiva, el capital cultural representa una teoría de la reproducción, es decir, se señala que las familias que poseen capital cultural tienen una ventaja comparativa que les ayuda a reproducir su posición socioeconómica privilegiada (Verdú et al., 2014). Sin embargo, Bourdieu diferenció tres concepciones del capital cultural: incorporado, institucionalizado y objetivo (Sieben y Lechner, 2019). De acuerdo con el autor francés, la primera forma tiene que ver con una disposición duradera de la mente y el cuerpo del individuo, la segunda concepción aparece cuando el capital cultural incorporado se reconoce en forma de una credencial académica y en un estado objetivado, el concepto se convierte en bienes culturales tales como libros, máquinas, etc. (Throsby, 1999).

Desde una perspectiva cuantitativa, algunos estudios han buscado medir el capital cultural basados en el interés de Bourdieu por una nueva formalización, ya que él entendió las limitaciones del análisis de regresión, pero el autor siempre buscó un marco-modelo geométrico general; estaba entusiasmado con la posibilidad de una futura integración de la regresión en el marco del análisis de datos geométricos (Sanders y Robson, 2009). Por ejemplo, Robson (2009) estima una regresión vía Mínimos Cuadrados Ordinarios (MCO) para medir el efecto de la inversión en capital cultural sobre el capital económico en adolescentes de Gran Bretaña. Los datos provienen de encuestas a partir del 11 de abril de 1970, recopilando información a los 5, 10, 26 y 29 años de edad de los participantes. Las variables que intervienen en los estudios son tres: capital económico (potencial de ingresos de los participantes de acuerdo a la familia), capital cultural (relación de los participantes entre ocio y tiempo destinado a actividades culturales como visitas a museos, zoológicos, conciertos, lectura y escritura) y capital social (afiliación a una red cultural por medio de una membresía como la afiliación a partidos políticos o asociaciones de residentes). La evidencia empírica muestra 
el sexo y los antecedentes familiares influyeron en el capital económico y algunas actividades del capital cultural tuvieron un efecto estadísticamente significativo y positivo en el capital económico. Por lo tanto, los resultados econométricos sugieren que existe una relación directa entre el capital cultural y el capital económico.

Empero, existen algunas críticas al concepto. Si se entiende que el capital cultural es una teoría de la acción individual iterativa con retroalimentación (Farkas, 2018), es decir, el individuo puede acceder al capital cultural por medio de relaciones sociales. Entonces, el capital cultural es limitado y su adquisición depende de la existencia de las relaciones (Simpson, 2017). Se puede concebir que el agente encargado de ofrecer las condiciones para desarrollar relaciones sociales propias que fomenten el capital cultural, es el Estado. Pero en pleno contexto neoliberal, el rol de la autoridad queda superado por los mecanismos de mercado, cada uno puede adquirir capital cultural según sus posibilidades financieras. Por lo que las oportunidades son limitadas.

En suma, en su concepción individualista, el concepto de capital cultural es similar a la propuesta del capital humano (Throsby, 1999). Aun así, el capital cultural sigue proveyendo de un marco explicativo de algunos procesos de selección y exclusión social a través de los sistemas educativos (Bracho, 1990). Entonces, las teorías persiguen propósitos diferentes y, por lo tanto, el enfoque del capital cultural no es una teoría alternativa a las cuestiones utilitaristas. Para Marginson (2017) es más fructífero ir a las raíces de la teoría del capital humano, entender la narrativa predeterminada en relación con su propio propósito, proporcionar una explicación universal de las relaciones entre educación-crecimiento, para poder encontrar sus defectos y virtudes. En otras palabras, lo que propone el autor es abatir los argumentos del capital humano desde su propio campo de acción. 


\section{La crítica de los economistas al enfoque del capital humano: revisión de la literatura empírica}

Existe una amplia literatura que estudia la relación capital humano-crecimiento económico, tanto estudios nacionales como internacionales. Monteils (2014) estudia el caso de Francia en dos periodos: 1891-1995 considerando la tasa de alfabetización contra la tasa de crecimiento del PIB y 1970-1996 basado en el nivel de educación (diplomas) y la tasa de crecimiento del producto. Por medio de pruebas econométricas, test de Granger, se concluye que no existe causalidad, es decir, significa que los rendimientos del capital humano están disminuyendo y el conocimiento producido por la educación no puede ser el motor del crecimiento económico autosostenido.

Jaoul (2004) también investiga el caso francés, pero lo compara con la evidencia de Alemania. Los datos que emplea es la evolución del PIB y el número de estudiantes en el periodo 1899-1937. Desde el enfoque de cointegración, los resultados estadísticos mostraron que no existe una relación de largo plazo entre las dos variables, en los dos países. Por lo que el autor procedió a estimar un modelo de Vectores Autorregresivos (VAR) para analizar la causalidad. En el caso de Alemania no existe una relación entre el PIB y el número total de estudiantes, pero en Francia la prueba arroja que la educación superior afecta al PIB.

También existen estudios para economías en vías de desarrollo, en ellos se muestran los mismos resultados divergentes desde la óptica de la teoría del capital humano. Por ejemplo, Kobzev et al. (2018) analizan la evidencia de India por medio de econometría. La prueba de causalidad se establece dentro del periodo 1975-2016 para medir el impacto de la educación primaria, secundaria y terciaria en el crecimiento económico (PIB per cápita). Los resultados indican que únicamente el sector terciario tiene una relación positiva con el crecimiento del producto. No obstante, esta evidencia sólo aplica principalmente a la población masculina.

Mientras Adawo (2011) estima un modelo doble logarítmico con base en las ideas del modelo de capital humano de G. Mankiw para 
Nigeria con datos anuales de 1970 a 2006. Las variables que considera la investigación son el PIB real, la formación de capital físico, el trabajo en general, la inscripción en la escuela primaria y la inscripción en la escuela secundaria, el trabajo de los graduados y el gasto total en salud. Los resultados estadísticos revelan que dos variables (escuelas secundarias e instituciones terciarias) frenan el crecimiento.

Finalmente, en Pakistán Khan et al. (2011) examinan la relación de largo plazo entre el gasto en educación y el crecimiento económico en el período 1980-2009. Mediante el uso de técnicas econométricas, cointegración y Vector de Corrección de Errores (VEC), se confirman los resultados esperados, la educación y el crecimiento del PIB se relacionan a largo periodo.

En suma, del análisis anterior se desprenden dos premisas. Primera, la mayoría de los estudios realizan pruebas econométricas por medio del análisis de series de tiempo para reducir las asimetrías que provocan el uso de la técnica MCO. Segunda, existen nulos estudios que indaguen la relación entre gasto educativo y crecimiento económico de México desde una perspectiva a posteriori, por lo tanto, se empleará dicha técnica estadística para el análisis de la economía mexicana en el periodo 1995-2016.

\section{Metodología econométrica y evidencia empírica}

\subsection{La prueba de causalidad en el sentido de Granger}

Se emplea el enfoque a priori, es decir, la econometría basada en series de tiempo. En particular, la evidencia empírica internacional emplea la prueba de Granger (1969), siendo el instrumento estadístico más común que determina la causalidad. Este test mide causalidad cuando una variable tiene influencia estadística sobre otra variable rezagada.

Para establecer el resultado general, en las Ecuaciones 1 y 2 se determina un modelo VAR de dos variables estacionarias y dos ecuaciones, 
cada expresión enunciada por los valores retardados de ambas variables y con errores con media cero:

Ecuación 1

$$
Y_{t}=\alpha_{0}+\alpha_{1} Y_{t-1}+\alpha_{2} Z_{t-1}+\alpha_{3} Y_{t-2}+\alpha_{4} Z_{t-2}+e_{t}
$$

Ecuación 2

$$
Z_{t}=\beta_{0}+\beta_{1} Y_{t-1}+\beta_{2} Z_{t-1}+\beta_{3} Y_{t-2}+\beta_{4} Z_{t-2}+e_{t}
$$

Para Wooldridge (2010), Z causa en el sentido de Granger a Y si el pronóstico no condicional de $Y_{t}$ dado $I_{t-1}$ es diferente de $Y_{t}$ dado $J_{t-1}$ :

Ecuación 3

$$
E\left(Y_{t} / I_{t-1}\right) \neq E\left(Y_{t} / J_{t-1}\right)
$$

Donde $I_{t-1}$ contiene a las variables $\mathrm{Y}$ y $\mathrm{Z}$ rezagadas en el tiempo t, mientras $J_{t-1}$ expresa información de $\mathrm{Y}$ en $\mathrm{t}-1$.

En la práctica, el software R estima la prueba de Granger por línea de comando con ayuda del package vars (Pfaff, 2008), lo cual implica una regresión vía MCO donde $\mathrm{Y}$ se explica sus propios términos rezagados y por otras variables (en este caso Z). Lo importante es entender que el estadístico de prueba se distribuye con la prueba $\mathrm{F}$, aunque se relación con la distribución normal limita su generalidad (Greene, 2003), y la hipótesis nula para la causalidad no instantánea busca que los términos rezagados de $\mathrm{Z}$ no pertenezcan a la regresión.

Ecuación 3

$$
H_{o}: \alpha_{i}=0 \quad \text { donde } i=1,2, \ldots, n
$$

Si el valor F calculado excede al valor F crítico en el nivel seleccionado de significancia, rechazamos la hipótesis nula, en cuyo caso los términos rezagados de $Z$ pertenecen a la regresión (Gujarati, 2009), por lo que $\mathrm{Z}$ causa en el sentido de Granger a $\mathrm{Y}$, entonces $\mathrm{Z}$ es un predictor 
útil de Y dadas otras variables de una regresión. Stock y Watson (2012) explican que dicho instrumento es sinónimo de predictibilidad más que de causalidad.

\subsection{Análisis empírico}

En la Gráfica 1 se muestran las tasas de crecimiento del gasto en educación desde mediados de la década de 1990, la tendencia de la variable analizada es decreciente en su modalidad Pública y Privada.

En la Gráfica 2 se presenta el comportamiento del gasto público por sector educativo, educación básica y educación superior, la variable en los dos sectores ha disminuido sustancialmente, hecho que disiente con el discurso del gobierno, cada vez son menores los recursos que se destinan a educación y la tendencia muestra claramente las prioridades del gobierno, la educación no es un sector importante para los gobiernos mexicanos recientes. De hecho, los esfuerzos han sido débiles ya que entre 2005 y 2010 el gasto por estudiante e institución educativa, en los niveles primario y secundario, aumento en 4\% para México, ritmo inferior al 17\% de los países de la OCDE (Cortés, 2015).

\section{Gráfica 1. Tasas de crecimiento del gasto federal en educación, privado y público (México, 1997-2016)}

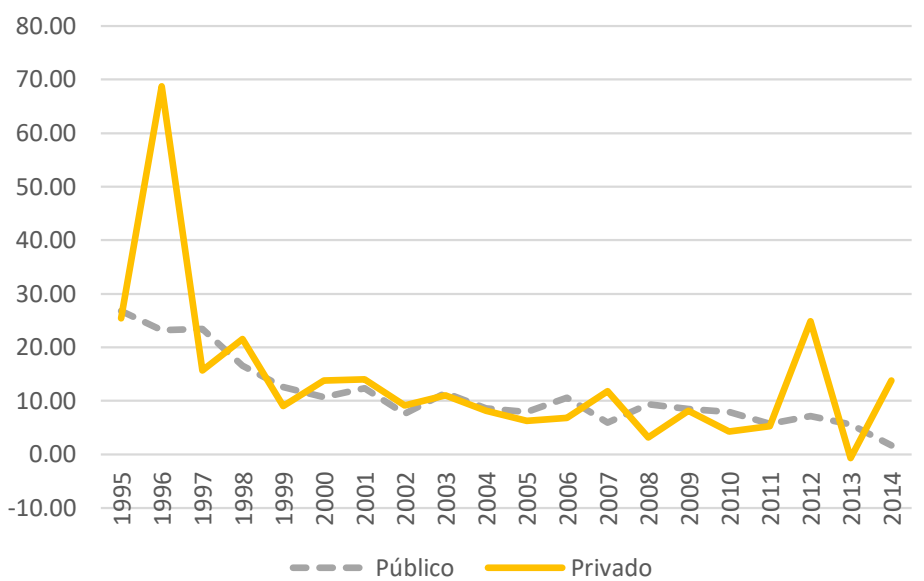

Fuente: elaboración propia con base en INEGI (2017). 


\section{Gráfica 2. Tasas de crecimiento del gasto público federal en educación (México, 1996-2016)}

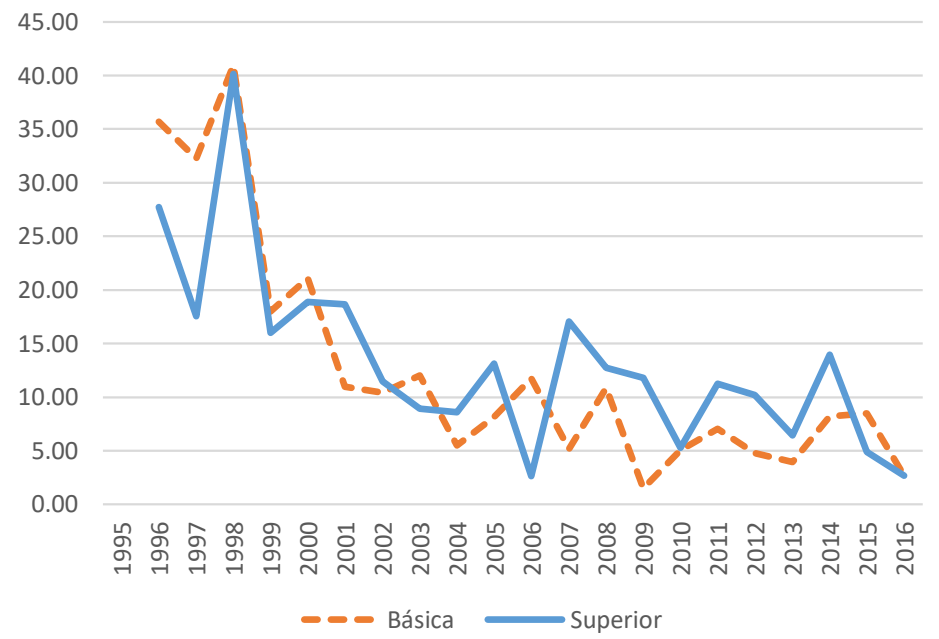

Fuente: elaboración propia con base en INEGI (2017).

\section{Gráfica 3 Tasas de crecimiento del PIB y gasto federal en educación (México, 1997-2016)}

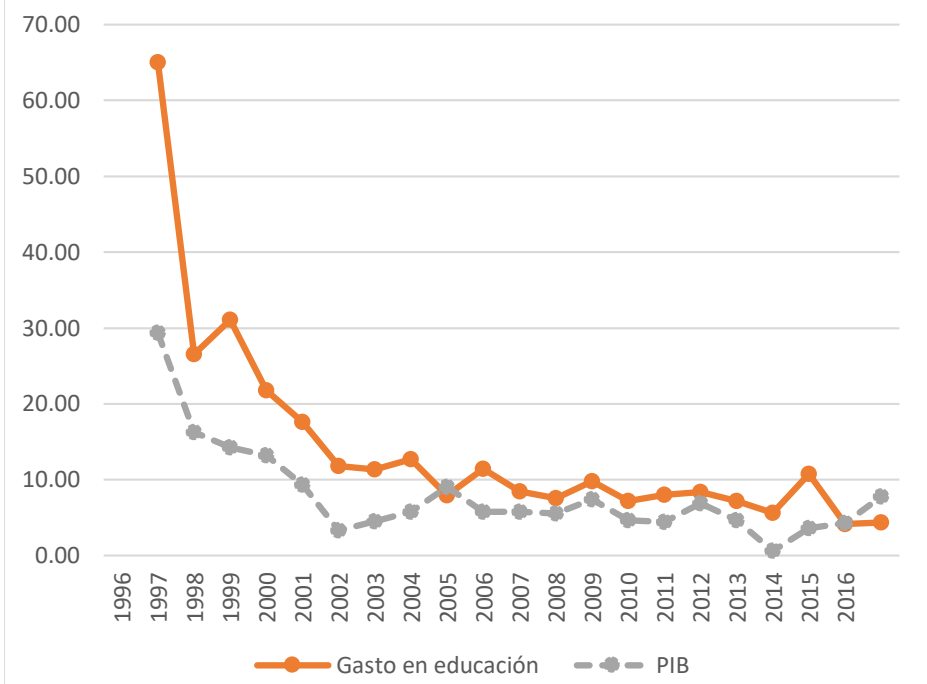

Fuente: elaboración propia con base en INEGI (2017). 
En la Gráfica 3 se indican las tasas de crecimiento del PIB y del gasto público en educación, éstas siguen un comportamiento similar pues las variables se ha estancado desde el nuevo milenio, es decir, la tasa promedio de crecimiento anual de 2000 a 2016 es de $5.49 \%$ y 9.07\% para el producto y el gasto respectivamente. Las dos variables aquí presentadas serán las analizadas en la siguiente sección por medio del enfoque econométrico a posteriori.

\subsection{El modelo para México}

Con el objetivo de determinar la prueba de causalidad se estiman tres pruebas de raíz unitaria a las dos variables en tasas de crecimiento, gasto en educación y PIB en precios de 2013. Las pruebas Dickey Fuller Aumentada (ADF) y Phillips Perron (PP) contrastan la hipótesis nula de raíz unitaria y la hipótesis alternativa de estacionariedad. La prueba Kwiatkowski, Phillips, Schmidt y Shin (KPSS) plantea como hipótesis nula estacionariedad y su hipótesis alternativa es comprobar raíz unitaria. En el Cuadro 1 se despliegan los resultados y se concluye que las variables son estacionarias.

\section{Cuadro 1. Pruebas de raíz unitaria y estacionariedad}

\begin{tabular}{|c|c|c|c|c|c|}
\hline Variable & Especificación & ADF & PP & KPSS & Orden \\
\hline \multirow{2}{*}{$\mathbf{G}$} & Constante & -7.7703 & -8.1740 & 0.5964 & $\mathrm{I}(0)$ \\
\cline { 2 - 6 } & Tendencia & -8.7128 & -8.6767 & 0.1781 & $\mathrm{I}(0)$ \\
\hline \multirow{2}{*}{ Y } & Constante & -5.9747 & -6.3888 & 0.5223 & $\mathrm{I}(0)$ \\
\cline { 2 - 6 } & Tendencia & -4.6636 & -4.8142 & 0.1605 & $\mathrm{I}(0)$ \\
\hline
\end{tabular}

Fuente: elaboración propia.

Cuadro 2. Prueba de causalidad en el sentido de Granger

\begin{tabular}{|c|c|c|c|c|c|c|}
\hline Hipótesis & Est./Prob. & $\mathbf{1}$ & $\mathbf{2}$ & $\mathbf{3}$ & $\mathbf{4}$ & $\mathbf{5}$ \\
\hline $\begin{array}{c}\text { G no causa } \\
\text { a Y }\end{array}$ & $\mathrm{F}$ & 2.3196 & 0.1612 & 0.6521 & 0.7528 & 1.0233 \\
\cline { 2 - 7 } & $\mathrm{Pr}$ & 0.1461 & 0.8527 & 0.598 & 0.5834 & 0.4902 \\
\hline $\begin{array}{c}\text { Y no causa } \\
\text { a G }\end{array}$ & $\mathrm{F}$ & 0.1581 & 1.9087 & 0.6461 & 0.1754 & 0.2947 \\
\cline { 2 - 7 } & $\operatorname{Pr}$ & 0.6959 & 0.1849 & 0.6015 & 0.9448 & 0.8969 \\
\hline
\end{tabular}

Fuente: elaboración propia. 
En el Cuadro 2 se indican los resultados de la prueba de causalidad en el sentido de Granger, después de cinco rezagos y en sus dos versiones, en otras palabras, se estima la dirección de causalidad del gasto al PIB y viceversa.

Los resultados revelan que existe independencia, o falta de causalidad, entre las variables analizadas, gasto en educación y crecimiento económico, es decir, la evidencia empírica que se muestra en esta investigación apunta a negar la validez práctica del enfoque del capital humano.

\section{Conclusiones}

Después del fin de la segunda guerra mundial una de las discusiones académicas es la relación educación-crecimiento. Los economistas, ahora postura divulgada por el BM, basan su respuesta en el enfoque del capital humano cuya lógica busca dotar de habilidades a los individuos por medio de la escolaridad y eso se traduce en puntos adicionales en el crecimiento del producto de un país. Los críticos a esta teoría utilitarista creen en la narrativa, pero es particular de cada región, por lo que la teoría neoclásica del capital humano no es universal. Existen otros elementos que los sociólogos han apuntado acertadamente, no sólo la economía es una variable que explique la riqueza de un país, también contribuye la cultura.

Desde una perspectiva empírica, los economistas han empleado el enfoque econométrico a priori, o de series de tiempo para la relación educación-crecimiento y las investigaciones en Francia, Alemania, India y Nigeria apuntan que no existe un efecto directo y solido entre la educación y el crecimiento.

En el caso de México, la investigación actual propone el mismo análisis a través de la prueba de causalidad en el sentido de Granger, la evidencia estadística sugiere que el gasto educativo no tiene impacto en el crecimiento y viceversa, esto se traduce en que la educación no es un motor para la riqueza del país porque es un sector no prioritario del Estado pues no existe una estrategia definida de largo plazo y un vínculo robusto y dinámico entre los egresados y el mercado laboral. 


\section{Bibliografía}

Adawo, M. (2011). "Has education (human capital) contributed to the economic growth of Nigeria?". Journal of Economics and International Finance, vol. 3, núm. 1, pp. 46-58.

Aghion. P. y P. Howitt (2009). The economics of growth. Massachusetts: The MIT press.

BM (2018). The Human Capital Project. Washington: Banco Mundial.

Barro, R. (1999). "Determinants of economic growth: implications of the global evidence for Chile". Revista Cuadernos de Economía, vol. 36, núm. 107, pp. 443-478.

Bourdieu, P. (1987). "Los tres estados del capital cultural". Revista Sociológica, vol. 2, núm. 5, pp. 11-17.

Bouznit, M. (2012). "Politiques educatives et croissance conomique: etude analytique et econometrique pour le cas de l'Algerie". Recuperado de http://www.enssea.net/enssea/moultakayat/2012/polpub/2012-34.pdf

Bracho, T. (1990). "Capital cultural: impacto en el rezago educativo". Revista Latinoamericana de Estudios Educativos, vol. 20, núm. 2, pp. 13-46.

Colorado, A. (2009). "El capital cultural y otros tipos de capital en la definición de las trayectorias escolares universitarias". X Congreso Nacional de Investigación Educativa.

Cortés, O. (2015). "Evaluación del gasto educativo en México". Centro de Estudios Sociales y de Opinión Pública, Reporte de investigación núm. 9.

De la Fuente, A. y A. Ciccone (2002). "Le capital humain dans une économie mondiale fondée sur la connaissance". Recuperado de https:// hip.lisboa.ucp.pt/edocs/cde/2003_2765.pdf

Delajara, M. et al. (2018) El México del 2018. Movilidad social para el bienestar. México: Centro de Estudios Espinosa Yglesias. 
Diop, A. (2011). Politique de recrutement des enseignants non fonctionnaires et qualité de l'éducation de base au Sénégal: quels enseignements vers l'Education Pour Tous (EPT)?. Tesis doctoral, Université de Bourgogne de Francia.

Domar, E. (1946). "Capital Expansion, Rate of Growth, and Employment". Revista Econometrica, vol. 14, núm. 2, pp. 137-147.

Farkas, G. (2018). "Family, Schooling, and Cultural Capital". En Schneider, B. (Ed.). Handbook of the Sociology of Education in the 21st Century. Suiza: Springer.

Galindo, M. y M. Méndez (2010). "Adam Smith on Education and economioc growth: direct and indirect effects". En Galindo, M. y C. Nardi. Issues in Economic Thought. Nueva York: Nova Science Publishers.

Granger, C. (1969). "Investigating causal relations by econometric models and cross-spectral methods". Econometrica, vol. 37, núm. 3, pp. 424-438.

Greene, W. (2003). Econometric analysis. Nueva Jersey: Pearson Educación.

Gujarati, D. y D. Porter (2009). Econometría. México: McGraw-Hill.

Hanushek E. y D. Kimko (2000). "Schooling, labor-force quality, and the growth of nations". The American Economic Review, vol. 90, núm. 5, pp. 1184-1208.

Hanushek, E. y L. Woessmann (2012). "The economics of international differences in educational achievement". Journal of Economic Growth, vol. NE, núm. 17, pp. 267-321.

Harrod, R. (1939). "An Essay in Dynamic Theory". The Economic Journal, vol. 49, núm. 193, pp. 14-33.

INEGI (2017). Anuario estadístico y geográfico de los Estados Unidos Mexicanos 2017. México: Instituto Nacional de Estadística y Geografía.

Jaoul, M. (2004). "Higher education, causality and growth: a comparison of France and Germany before the Second World War". Compa- 
re: A Journal of Comparative and International Education, vol. 34, núm. 1, pp. 117-133.

Khan, Z. et al. (2011). "Relationship between Education and Economic Growth in Pakistan: A time series analysis". Journal of International Academic Research, vol.11, núm.1, pp. 27-32.

Kobzev, S. et al. (2018). "The Impact of Education on Economic Growth: The Case of India". Acta Universitatis Agriculturae et Silviculturae Mendelianae Brunensis, vol. 66, núm. 1, pp. 253-262.

Lauder, H. (2015). "Human capital theory, the power of transnational companies and a political response in relation to education and economic development". Compare: A Journal of Comparative and International Education, vol. 45, núm. 3, pp. 490-493.

Laval, C. (2004). La escuela no es una empresa. Madrid: Paidós.

Mankiw, G. et al. (1992). "A Contribution to the Empirics of Economic Growth". The Quarterly Journal of Economics, vol. 107, núm. 2, pp. 407-437.

Marcenaro, Óscar. y L. López (2014). "El efecto del capital cultural sobre el rendimiento educativo diferencial por género". Recuperado de https://idus.us.es/xmlui/bitstream/handle/11441/41053/Pages\%20 from $\% 20$ Investigacion_Genero_14-2-12.pdf?sequence $=1$

Marginson, S. (2017). "Limitations of human capital theory". Studies in Higher Education, vol. 44, núm. 2, pp. 287-301.

Mayia, F. et al. (2004). "Human capital theory: implications for human resource development". Journal Human Resource Development International, vol. 7, núm. 4, pp. 545-551.

Monteils, M. (2014). "The analysis of the relation between education and economic growth". Compare: A Journal of Comparative and International Education, vol. 34, núm. 1, pp. 103-115.

OCDE (2018). A Broken Social Elevator? How to Promote Social Mobility. Overview and main findings. Paris: OCDE. 
Okacha, R. (2015). Capital humain, education et croissance économique. Tesis de doctoral, Université Abou Bekr Belkaid de Argelia .

Pfaff, B. (2008). Analysis of integrated and cointegrated time series with $R$. Nueva York: Springer.

Piketty, T. (2014). El capital en el siglo XXI. Buenos Aires: Fondo de Cultura Económica.

Rajimon, J. (2010). “La economía y la función de producción en educación”. Revista Visión de Futuro, vol. 13, núm. 1, pp. 201-215.

Robson, K. (2009). "Teenage Time Use as Investment in Cultural Capital". En Robson K. y C. Sanders (eds.). Quantifying Theory: Pierre Bourdieu. Toronto: Springer Netherlands.

Sanders, C. y K. Robson (2009). "Introduction: Approaches to Quantifying Bourdieu”. En Robson, K. y C. Sanders (eds.). Quantifying Theory: Pierre Bourdieu. Toronto: Springer Netherlands.

Sieben, S. y C. Lechner (2019). "Measuring cultural capital through the number of books in the household". Journal Measurement Instruments for the Social Sciences, vol. 2, núm. 1, pp. 1-6.

Simpson, C. (2017). "The acquisition of cultural capital. Gabriellla's Story”. En Simpson, C. Experiences of Women of Color in an Elite US Public School. Suiza: Springer.

Solow, R. (1956). "A Contribution to the Theory of Economic Growth". The Quarterly Journal of Economics, vol. 70, núm. 1, pp. 65-94.

Stock, J. y M. Watson (2012). Introducción a la Econometría. Madrid: Pearson Educación.

Throsby, D. (1999). “Capital cultural”. Journal of Cultural Economics, vol. 23, núm. 3, pp. 3-12.

Valdenaire, M. (2011). “Essais en économie de l'éducation”. École des Hautes Études en Sciences Sociales en Francia (Tesis de doctorado). 
Verdú, A. et al. (2014). "Capital cultural y social: sus efectos en el conocimiento financiero según PISA 2012". Ministerio de Educación, Cultura y Deporte, documento de trabajo.

Weil, D. (2006). Crecimiento económico. Madrid: Pearson Educación.

Wooldridge, J. (2010). Introducción a la econometría Un enfoque moderno. México: Cengage Learning Editores.

\section{Anexo estadístico}

\begin{tabular}{|c|c|c|}
\hline \multicolumn{3}{|c|}{ Pruebas de Raíz Unitaria (Variable G) } \\
\hline ADF & PP & KPSS \\
\hline $\begin{array}{l}\text { Value of test-statistic is: } \\
-7.770334 .7389 \\
\text { Critical values for test } \\
\text { statistics: } 1 \text { pct } 5 \text { pct } 10 \text { pct } \\
\text { tau2 }-3.75-3.00-2.63 \\
\text { phi1 } 7.885 .184 .12\end{array}$ & $\begin{array}{l}\text { Value of test-statistic, type: } \\
\text { Z-tau is: }-8.174 \\
\text { aux. Z statistics } \\
\text { Z-tau-mu } 3.8241 \\
\text { Critical values for } Z \text { statistics: } \\
\text { 1pct } 5 \text { pct } 10 \text { pct } \\
\text { critical values }-3.806575 \\
-3.0199-2.6502\end{array}$ & $\begin{array}{l}\text { Value of test-statistic is: } \\
0.5964 \\
\text { Critical value for a signifi- } \\
\text { cance level of: } 10 \mathrm{pct} 5 \mathrm{pct} \\
2.5 \mathrm{pct} 1 \mathrm{pct} \\
\text { critical values } 0.3470 .463 \\
0.5740 .739\end{array}$ \\
\hline $\begin{array}{l}\text { Value of test-statistic is: } \\
-8.712836 .256247 .8426 \\
\text { Critical values for test } \\
\text { statistics: } 1 \text { pct } 5 \text { pct } 10 \text { pct } \\
\text { tau3 }-4.38-3.60-3.24 \\
\text { phi2 } 8.215 .684 .67 \\
\text { phi3 } 10.617 .245 .91\end{array}$ & $\begin{array}{l}\text { Value of test-statistic, type: } \\
\text { Z-tau is: }-8.6767 \\
\text { aux. Z statistics } \\
\text { Z-tau-mu } 5.2644 \\
\text { Z-tau-beta }-2.9764 \\
\text { Critical values for Z statistics: } \\
\text { 1pct } 5 \text { pct } 10 \text { pct } \\
\text { critical values }-4.50005 \\
-3.659125-3.26775\end{array}$ & $\begin{array}{l}\text { Value of test-statistic is: } \\
0.1781 \\
\text { Critical value for a signifi- } \\
\text { cance level of: } 10 \text { pct } 5 \text { pct } \\
2.5 p c t 1 \text { pct } \\
\text { critical values } 0.1190 .146 \\
0.1760 .216\end{array}$ \\
\hline
\end{tabular}




\begin{tabular}{|c|c|c|}
\hline \multicolumn{3}{|c|}{ Pruebas de Raíz Unitaria (Variable Y) } \\
\hline ADF & PP & KPSS \\
\hline $\begin{array}{l}\text { Value of test-statistic is: } \\
-5.974720 .0205 \\
\text { Critical values for test } \\
\text { statistics: } 1 \text { pct } 5 \text { pct } 10 \text { pct } \\
\text { tau2 }-3.75-3.00-2.63 \\
\text { phi1 } 7.885 .184 .12\end{array}$ & $\begin{array}{l}\text { Value of test-statistic, type: } \\
\text { Z-tau is: }-6.3888 \\
\text { aux. Z statistics } \\
\text { Z-tau-mu } 3.567 \\
\text { Critical values for Z statistics: } \\
\text { 1pct 5pct } 10 \text { pct } \\
\text { critical values }-3.806575 \\
-3.0199-2.6502\end{array}$ & $\begin{array}{l}\text { Value of test-statistic is: } \\
0.5223 \\
\text { Critical value for a signifi- } \\
\text { cance level of: } 10 \text { pct } 5 p c t \\
2.5 p c t \text { pct } \\
\text { critical values } 0.3470 .463 \\
0.5740 .739\end{array}$ \\
\hline $\begin{array}{l}\text { Value of test-statistic is: } \\
-4.663613 .223217 .7144 \\
\text { Critical values for test } \\
\text { statistics: } 1 \text { pct } 5 \text { pct } 10 \text { pct } \\
\text { tau3 }-4.38-3.60-3.24 \\
\text { phi2 } 8.215 .684 .67 \\
\text { phi3 } 10.617 .245 .91\end{array}$ & $\begin{array}{l}\text { Value of test-statistic, type: } \\
\text { Z-tau is: }-4.8142 \\
\text { aux. Z statistics } \\
\text { Z-tau-mu } 3.6489 \\
\text { Z-tau-beta }-0.6701 \\
\text { Critical values for Z statistics: } \\
\text { 1pct 5pct } 10 \text { pct } \\
\text { critical values }-4.50005 \\
-3.659125-3.26775\end{array}$ & $\begin{array}{l}\text { Value of test-statistic is: } \\
0.1605 \\
\text { Critical value for a signifi- } \\
\text { cance level of: } 10 \mathrm{pct} 5 \mathrm{pct} \\
2.5 \mathrm{pct} 1 \mathrm{pc} \\
\text { critical values } 0.1190 .146 \\
0.1760 .216\end{array}$ \\
\hline
\end{tabular}




\begin{tabular}{|c|c|c|}
\hline \multicolumn{3}{|c|}{ Prueba de Causalidad en el sentido de Granger } \\
\hline Rezago & G no causa a $Y$ & Y no causa a $G$ \\
\hline 1 & $\begin{array}{l}\text { Model 1: G } \sim \operatorname{Lags}(G, 1: 1)+ \\
\text { Lags(Y, 1:1) } \\
\text { Model 2: G } \sim \operatorname{Lags}(G, 1: 1) \\
\text { Res.Df Df F Pr }(>F) \\
117 \\
218-12.31960 .1461\end{array}$ & $\begin{array}{l}\text { Model 1: Y } \sim \operatorname{Lags}(Y, 1: 1)+ \\
\text { Lags(G, 1:1) } \\
\text { Model 2: } Y \sim \operatorname{Lags}(Y, 1: 1) \\
\text { Res.Df Df F Pr }(>F) \\
117 \\
218-10.15810 .6959\end{array}$ \\
\hline 2 & $\begin{array}{l}\text { Model 1: G } \sim \operatorname{Lags}(G, 1: 2)+ \\
\text { Lags(Y, 1:2) } \\
\text { Model 2: } \mathrm{G} \sim \operatorname{Lags}(\mathrm{G}, 1: 2) \\
\text { Res.Df Df F Pr }(>\mathrm{F}) \\
114 \\
216-20.16120 .8527\end{array}$ & $\begin{array}{l}\text { Model 1: Y } \sim \operatorname{Lags}(Y, 1: 2)+ \\
\text { Lags(G, 1:2) } \\
\text { Model 2: Y } \sim \operatorname{Lags}(Y, 1: 2) \\
\text { Res.Df Df F Pr }(>F) \\
114 \\
216-21.90870 .1849\end{array}$ \\
\hline 3 & $\begin{array}{l}\text { Model 1: G } \sim \operatorname{Lags}(G, 1: 3)+ \\
\text { Lags(Y, 1:3) } \\
\text { Model 2: G } \sim \operatorname{Lags}(G, 1: 3) \\
\text { Res.Df Df F Pr(>F) } \\
111 \\
214-30.65210 .598 \\
\end{array}$ & $\begin{array}{l}\text { Model 1: } \mathrm{Y} \sim \operatorname{Lags}(\mathrm{Y}, 1: 3)+ \\
\operatorname{Lags}(\mathrm{G}, 1: 3) \\
\text { Model 2: } \mathrm{Y} \sim \operatorname{Lags}(\mathrm{Y}, 1: 3) \\
\text { Res.Df Df F } \operatorname{Pr}(>\mathrm{F}) \\
111 \\
214-30.64610 .6015\end{array}$ \\
\hline 4 & $\begin{array}{l}\text { Model 1: G Lags }(G, 1: 4)+ \\
\text { Lags(Y, 1:4) } \\
\text { Model 2: G } \sim \operatorname{Lags}(G, 1: 4) \\
\text { Res.Df Df F Pr }(>F) \\
18 \\
212-40.75280 .5834\end{array}$ & $\begin{array}{l}\text { Model 1: Y Lags }(Y, 1: 4)+ \\
\text { Lags }(G, 1: 4) \\
\text { Model 2: } Y \sim \operatorname{Lags}(Y, 1: 4) \\
\text { Res.Df Df F Pr }(>F) \\
18 \\
212-40.17540 .9448\end{array}$ \\
\hline 5 & $\begin{array}{l}\text { Model 1: G } \sim \operatorname{Lags}(G, 1: 5)+ \\
\text { Lags }(Y, 1: 5) \\
\text { Model 2: } \mathrm{G} \sim \operatorname{Lags}(G, 1: 5) \\
\text { Res.Df Df F Pr }(>F) \\
15 \\
210-51.02330 .4902\end{array}$ & $\begin{array}{l}\text { Model 1: Y } \sim \operatorname{Lags}(Y, 1: 5)+ \\
\text { Lags }(G, 1: 5) \\
\text { Model } 2: Y \sim \operatorname{Lags}(Y, 1: 5) \\
\text { Res.Df Df F } \operatorname{Pr}(>F) \\
15 \\
210-50.29470 .8969\end{array}$ \\
\hline
\end{tabular}


